\title{
Stand und Potenziale der Versorgungsforschung: Die Perspektive der Akteure in Bayern
}

\section{Status Quo and Potential of Health Services Research: The Perspective of Bavarian Actors}

Autoren

Institut
A. Hollederer, S. Voigtländer, M. Wildner, A. Zapf, A. Zellner

Bayerisches Landesamt für Gesundheit und Lebensmittelsicherheit (LGL), Nürnberg

\section{Schlüsselwörter \\ - Versorgungsforschung \\ - Gesundheitspolitik \\ - Bayern \\ - Befragung}

Key words

- health services research

- health policy

- Bavaria

- survey

\section{Bibliografie}

DOI http://dx.doi.org/

10.1055/s-0034-1367030

Online-Publikation: 6.5.2014

Gesundheitswesen 2015;

77: 186-192

(c) Georg Thieme Verlag KG

Stuttgart · New York

ISSN 0941-3790

\section{Korrespondenzadresse}

\section{Dr. Sven Voigtländer}

Bayerisches Landesamt für Gesundheit und Lebens-

mittelsicherheit

Bayerisches Haus der

Gesundheit

Sachgebiet (GE 6):

Versorgungsqualität,

Gesundheitsökonomie,

Gesundheitssystemanalyse

Schweinauer Hauptstraße 80

90441 Nürnberg

sven.voigtlaender@|gl.bayern.de

\section{License term}

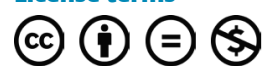

\section{Zusammenfassung}

$\nabla$

Hintergrund: Der Bayerische Landtag hat im Jahr 2011 beschlossen, die Versorgungsforschung in Bayern weiterzuentwickeln und die Kompetenzen der Wissenschaft in Form einer Landesarbeitsgemeinschaft zu bündeln und mit weiteren Beteiligten zu vernetzen. Die Gründung dieser Landesarbeitsgemeinschaft Gesundheitsversorgungsforschung (LAGeV) erfolgte mit relevanten Mitgliedern aus der Wissenschaft, Versorgungspraxis und Politik im Jahr 2012. Ziel der vorliegenden Untersuchung ist es, aus Sicht dieser Akteure den Stand der Versorgungsforschung in Bayern zu analysieren sowie die Entwicklungspotenziale und Einflussfaktoren zu identifizieren. Methodik: Nach der konstituierenden Sitzung der LAGeV wurde ein teilstandardisierter Fragebogen an alle 36 Mitgliedspersonen aus 28 Organisationen versandt. Die Items umfassen Angaben zum Hintergrund des Befragten sowie zum Stand, zu den Zukunftsthemen und Potenzialen der Versorgungsforschung in Bayern.

Ergebnisse: 27 Mitglieder nahmen an der Befragung teil, was einer Rücklaufquote von $75,0 \%$ entspricht. Die Zufriedenheit der Akteure mit dem Stand der Versorgungsforschung ist vergleichsweise gering; dies gilt insbesondere hinsichtlich der Wirksamkeit in der Politikberatung. Die Wissenschaftler und Leistungserbringer sind auch mit den Rahmenbedingungen besonders unzufrieden. Als das wichtigste Zukunftsthema der Versorgungsforschung wird die Schnittstellen- und Vernetzungsforschung erachtet. Es folgen die Themen innovative Versorgungskonzepte, Versorgung von multimorbiden Patienten bzw. chronisch Kranken sowie Evaluation von Innovationen, Prozessen und Verfahren. Potenziale zur Weiterentwicklung der Versorgungsforschung und damit zur Optimierung der Versorgung bestehen aus Sicht der Befragten vor allem in der Beseitigung von bisher hemmenden Faktoren. Konkrete Ansatzpunkte werden in einer

\section{Abstract \\ $\nabla$}

Background: In 2011, the Bavarian Parliament decided to advance health services research (HSR) in Bavaria by bundling scientific competencies in a State Working Group and integrating other actors in it. The establishment of such a State Working Group "Health Services Research" (LAGeV) together with members from science, health care and politics followed in 2012. The objective of this study is to identify the status quo of HSR in Bavaria including its determinants and potential for development based on the actors' perspective.

Methods: After the inaugural meeting a semistructured questionnaire was sent to all 36 members from 28 organisations. Items comprise information on the respondent's background as well as status quo, future topics and potential for development of HSR in Bavaria.

Results: 27 members took part in the survey, resulting in a response rate of $75.0 \%$. Satisfaction of actors with the status quo of HSR is rather low, especially regarding the effectiveness of policy advice. Researchers and health care providers are also not much satisfied with the HSR environment. For the future of HSR, respondents prioritise the topics interface and networking research, followed by innovative care concepts, care for patients with multiple or chronic conditions as well as evaluation of innovations, processes and technologies. Potential for development and thus improvement of care is primarily seen in the abolishment of existing constraints by an overall HSR concept (including selective research promotion), networking and cooperation, research funding as well as improving the interface between politics and science. Respondents assess the benefit of an increased networking within the LAGeV as high.

Conclusions: Status quo of HSR in Bavaria is not very satisfactory. The survey reveals important constraints as well as promoting factors based on 
Rahmenkonzeption mit gezielter Forschungsförderung, in Vernetzung und Kooperation, Forschungsfinanzierung sowie einer Verbesserung der Schnittstelle Politik-Versorgungsforschung gesehen. Der Mehrwert einer stärkeren Vernetzung im Rahmen der LAGeV wird als hoch eingeschätzt.

Schlussfolgerungen: Der Stand der Versorgungsforschung in Bayern ist nur bedingt zufriedenstellend. Die Erhebung zeigt wichtige Hemm- und Förderfaktoren aus der Perspektive der unterschiedlichen Akteursgruppen auf. Sie priorisiert die $\mathrm{Zu}$ kunftsthemen in der Versorgungsforschung und identifiziert Ansatzpunkte für die Landesarbeitsgemeinschaft. Diese Befunde können für eine Weiterentwicklung der Versorgungsforschung in Bayern und darüber hinaus genutzt werden. the viewpoints of different groups of actors. It also prioritises future HSR topics and identifies potential for development, which are important for the LAGeV. The findings can be used for advancing HSR in Bavaria and beyond.

\section{Hintergrund}

$\nabla$

Angesichts der erheblichen Herausforderungen im Gesundheitswesen weist der Sachverständigenrat (SVR) zur Begutachtung der Entwicklung im Gesundheitswesen im Sondergutachten 2012 darauf hin, dass „eine gezielte Förderung von Versorgungsinnovationen in Verbindung mit einer entsprechenden Versorgungsforschung unabdingbar erscheint" $[1$, S. 396]. Bereits im Gutachten 2000/2001 verwies der SVR „auf den notwendigen Ausbau einer angemessenen Qualitäts- und Versorgungsforschung in Deutschland“ [2, S. 105] und mahnte seitdem mehrmals die Umsetzung an [3]. Für die bedarfsgerechte Steuerung des komplexen Gesundheitssystems werden umfassende wissenschaftliche Erkenntnisse der Gesundheitsversorgungsforschung benötigt. Es gilt, Unter-, Über- und Fehlversorgung zu vermeiden. Der Begriff „Versorgungsforschung“ bezieht sich allerdings nach Raspe et al. [4, S. 11] „weniger auf ein umgrenztes oder gar spezifisches Arsenal von wissenschaftlichen Methoden, Techniken und Instrumenten, sondern vielmehr auf:

- ein besonderes Problemfeld als Gegenstand: die gesundheitliche oder enger noch: medizinische Versorgung unserer Bevölkerung,

- eine bestimmte Frageperspektive, die auf die Organisation, Regulierung und Verbesserung dieser Versorgung zielt, und

- ein multi- und transprofessionelles Praxisfeld: die wissenschaftlich fundierte Informierung und Beratung von Personen und Einrichtungen mit Steuerungsfunktionen im Gesundheitswesen".

Nach der internationalen Grundlagendefinition von „Health Services Research" von Lohr und Steinwachs [5] wird Versorgungsforschung als multidisziplinäres Gebiet verstanden, das wissenschaftlich untersucht, wie soziale Faktoren, Finanzierungssysteme, Organisationsstrukturen und -prozesse, Gesundheitstechnologien und personale Verhaltensweisen die Zugangswege, Qualität und Kosten in der Gesundheitsversorgung und letztlich die Gesundheit und Wohlbefinden beeinflussen. Laut Pfaff [6] beschreibt und erklärt Versorgungsforschung kausal die Kranken- und Gesundheitsversorgung und ihre Rahmenbedingungen, trägt zur Entwicklung wissenschaftlich fundierter Versorgungskonzepte bei, erforscht begleitend die Umsetzung neuer Versorgungskonzepte und evaluiert die Wirksamkeit von Versorgungsstrukturen und -prozessen unter Alltagsbedingungen. Die Versorgungsforschung hat sich auf Basis dieser breit angelegten und multidisziplinären Konzeption in Deutschland mittlerweile etabliert, aber ihr Potenzial noch nicht ausgeschöpft. Die zentralen Herausforderungen, denen sich die deutsche Versorgungsforschung aus Sicht ihrer Protagonisten stellen muss, sind dabei grundlegender Natur und betreffen „die Zersplitterung der Akteure, die ungenügende Grundlagenorientierung der Forschung und die noch immer gegebene ungenügende Finanzierung“ [7, S. 11]. Notwendig sind mehr koordinierende Strukturen sowie die Koordination und Netzwerkbildung nicht nur innerhalb der Universitäten, sondern auch in überregionalen Strukturen (ebd.).

Über diese Situation hat der Bayerische Landtag am 17.03.2011 beraten und beschlossen, die Versorgungsforschung in Bayern weiterzuentwickeln und die Kompetenzen der Wissenschaft und weiterer Beteiligter im Gesundheitswesen in Form einer Landesarbeitsgemeinschaft einzubeziehen und zu vernetzen [8]. Die Einrichtung einer solchen Stelle zur Gesundheitsversorgungsforschung wurde als wichtige Komponente der bayerischen Gesundheitsstrategie mit der Regierungserklärung vom 20.10.2011 bekräftigt. Die Gründung einer entsprechenden Landesarbeitsgemeinschaft Gesundheitsversorgungsforschung (LA$\mathrm{GeV}$ ) erfolgte am 24.09.2012. Die Geschäftsstelle ist am Bayerischen Landesamt für Gesundheit und Lebensmittelsicherheit (LGL) angesiedelt. Die LAGeV nimmt als unabhängige Plattform der Versorgungsforschung seitdem eine koordinierende und moderierende Funktion an der Schnittstelle von Wissenschaft, Versorgungspraxis und Politik ein. Zu den Mitgliedsorganisationen zählen alle bayerischen Universitäten mit ihren medizinischen Fakultäten und Lehrstühlen für Gesundheitsökonomie sowie Forschungsinstitute und Fachhochschulen ( $\bullet$ Tab. 1). Darüber hinaus sind relevante Verbände, Vereine und Körperschaften sowie Politikfelder übergreifend die zuständigen Landesministerien - für Gesundheit und Pflege; Arbeit und Soziales, Familie und Integration; Bildung und Kultus, Wissenschaft und Kunst - unter Federführung des Staatsministeriums für Gesundheit und Pflege (StMGP) vertreten. Die Ziele der LAGeV sind:

- die Versorgungsforschung in Bayern weiterzuentwickeln,

- die Gesundheitsversorgung im Hinblick auf Bedarfsgerechtigkeit, Effizienz und Effektivität zu optimieren,

- prioritäre Gesundheitsprobleme zu identifizieren,

- Lösungsansätze in der Gesundheitsversorgung zu erarbeiten,

- die bürger- und patientenorientierte Gesundheitsversorgung zu optimieren,

- die Versorgungsqualität zu sichern und weiterzuentwickeln,

- regionalspezifische Versorgungsbedarfe zu identifizieren, um in der Bedarfsplanung einen guten Zugang zur Versorgung sicherstellen zu können,

- Gender Mainstreaming zu unterstützen,

- die Kooperation der Gesundheitsberufe zu intensivieren und

- die sektorenübergreifende Zusammenarbeit in der ambulanten und stationären Leistungserbringung zu verbessern. 
Tab. 1 Mitgliedsorganisationen der Bayerischen Landesarbeitsgemeinschaft Gesundheitsversorgungsforschung (LAGeV).

1. Bayerisches Staatsministerium für Gesundheit und Pflege

a. Abteilung 2 Krankenhausversorgung

b. Abteilung 3 Gesundheitspolitik, ambulante Versorgung, Krankenversicherung

c. Patientenbeauftragter

d. Landesarzt

2. Bayerisches Staatsministerium für Arbeit und Soziales, Familie und Integration

3. Bayerisches Staatsministerium für Bildung und Kultur, Wissenschaft und Kunst

4. Bayerisches Landesamt für Gesundheit und Lebensmittelsicherheit

5. Bayerische Landesärztekammer

6. Bayerische Landeszahnärztekammer

7. Bayerische Landeskammer der Psychologischen Psychotherapeuten und der Kinder- und Jugendlichenpsychotherapeuten

8. Kassenärztliche Vereinigung Bayerns

9. Bayerische Krankenhausgesellschaft e. V.

10. Bayerische Fachkrankenhäuser für Psychiatrie, Psychotherapie und Psychosomatik

11. AOK Bayern

12. Betriebskrankenkassen, Landesverband Bayern

13. Verband der Ersatzkassen e. V., Landesvertretung Bayern

14. Deutsche Rentenversicherung Bayern Süd

15. Kooperationsverbund Qualitätssicherung durch Klinische Krebsregister

16. Medizinischer Dienst der Krankenversicherung in Bayern

17. Verband der forschenden Pharma-Unternehmen

18. Universität Augsburg, Interdisziplinäres Zentrum für Gesundheitsforschung

19. Universität Bayreuth, Institut für Medizinmanagement und Gesundheitswissenschaften

20. Ludwig-Maximilians-Universität München

a. Institut und Poliklinik für Arbeits-, Sozial- und Umweltmedizin

b. Institut für Medizinische Informationsverarbeitung, Biometrie und Epidemiologie

21. Technische Universität München, Institut für Allgemeinmedizin

22. Friedrich-Alexander-Universität Erlangen-Nürnberg

a. Lehrstuhl für Gesundheitsmanagement

b. Institut und Poliklinik für Arbeits-, Sozial- und Umweltmedizin

c. Interdisziplinäres Zentrum für Public Health

d. Zentrum für Medizinische Versorgungsforschung der Psychiatrischen und Psychotherapeutischen Klinik

23. Julius-Maximilians-Universität Würzburg, Institut für Klinische Epidemiologie und Biometrie

24. Universität Regensburg, Institut für Epidemiologie und Präventivmedizin

25. Universität der Bundeswehr München, Institut für Management öffentlicher Aufgaben und Forschungszentrum für Management im Gesundheitswesen

26. Katholische Stiftungsfachhochschule München, Lehrstuhl für gerontologische Pflegewissenschaft und Qualitätsmanagement

27. Hochschule Regensburg, Fakultät Angewandte Sozialwissenschaften

28. Helmholtz Zentrum München, Institut für Gesundheitsökonomie und Management im Gesundheitswesen

Vor dem Hintergrund der Gründung der LAGeV hat die vorliegende Untersuchung das Ziel, den Stand der Versorgungsforschung in Bayern zu analysieren, die Entwicklungspotenziale und Einflussfaktoren zu identifizieren, konkrete Empfehlungen und Schwerpunktsetzungen für die LAGeV sowie die Möglichkeiten der Mitarbeit der einzelnen Mitglieder zu eruieren. Die Ergebnisse der Vollerhebung bei den LAGeV-Mitgliedern dienen der gemeinsamen Entwicklung einer Strategie zur Gesundheitsversorgungsforschung, in der die globalen Ziele operationalisiert sowie erste Prioritäten, Umsetzungsschritte, Methoden und Arbeitsformen festgelegt werden.

\section{Methodik}

\section{Erhebungsinstrument}

Die Bestandsanalyse überprüft zum einen nationale Erkenntnisse zum Stand der Versorgungsforschung auf ihre Gültigkeit für das Land Bayern. Sie hat zum anderen explorativen Charakter. Aus diesem Grund wird ein teilstandardisierter Fragebogen eingesetzt. Die offenen Fragen geben Gelegenheit, wesentliche Hemm- und Förderfaktoren der Versorgungsforschung in Bayern aus Expertensicht zu identifizieren und konkrete Empfehlungen für die Weiterentwicklung vorzunehmen. Das Erhebungsinstrument umfasst insgesamt 17 Items mit Angaben:

- zum Hintergrund des Befragten und seiner Organisation (4 Items),

- zum Stand, zu den Zukunftsthemen und Potenzialen der Versorgungsforschung in Bayern (7),

- zur konkreten Kooperation in der LAGeV (5) und bietet

- die Möglichkeit einer freien, themenunabhängigen Rückmeldung (1).

Die Items zur Interviewperson und seiner Organisation beinhalten Fragen zur Akteursgruppe sowie der Durchführung von Versorgungsforschung einschließlich der konkreten Teilgebiete basierend auf Pfaff [6]. Hinsichtlich der Versorgungsforschung in Bayern beziehen sich die Items auf die Zufriedenheit der Befragten mit zentralen Aspekten (11-stufige Likert-Skala von „ganz und gar unzufrieden“ bis „ganz und gar zufrieden“ bezüglich Rahmenbedingungen, Output und Wirksamkeit in der Politikberatung), die für die Versorgungsforschung in Bayern hemmenden und fördernden Faktoren (offene Fragen mit jeweils bis zu 3 Nennungen), die möglichen Zukunftsthemen für die Versorgungsforschung in Bayern sowie die Entwicklungspotenziale und die diesbezüglichen Chancen und Risiken (offene Fragen mit jeweils bis zu 3 Nennungen). Die Liste der möglichen Zukunftsthemen umfasst 21 verschiedene Themen, die die Befragten in ihrer Wichtigkeit bewerten können (7-stufige Likert-Skala von „ganz und gar unwichtig“ bis „ganz und gar wichtig“). Dabei können die Befragten bis zu 3 weitere Zukunftsthemen ergänzen. Die Auswahl der vorgegebenen Themen orientiert sich zum Großteil an den in der Literatur genannten Zukunftsthemen der Versorgungsforschung, vor allem von Grenz-Fahrenholtz et al. [9], Glaeske et al. [10], Pfaff und Kaiser [11] sowie Schubert et al. [12]. Die abgefragten Themen decken inhaltlich ein weites Spektrum in der Versorgungsforschung ab. Die Items zur LAGeV erfassen die Erwartung der Mitglieder an den damit verbundenen Mehrwert, ihre Bereitschaft zur aktiven Teilnahme sowie potenzielle Beiträge auf einer Informationsplattform.

\section{Durchführung der Befragung}

Die anonyme Befragung richtete sich an alle Mitglieder der LAGeV mit insgesamt 36 Personen aus 28 Mitgliedsorganisationen ( $\odot$ Tab. 1). Der postalische Versand der Fragebögen erfolgte am 28.11.2012 unter Beilage eines frankierten Rückumschlages. Am 20.12.2012 wurde an alle potenziellen Teilnehmer per Email ein Erinnerungsschreiben versandt.

\section{Datenauswertung}

Die geschlossenen Fragen wurden zunächst auf fehlende Werte überprüft und hinsichtlich ihrer Häufigkeitsverteilungen ein- 
zeln ausgewertet. Danach wurden Kreuztabellen berechnet, um nach bestimmten Merkmalen stratifizierte Häufigkeitsverteilungen untersuchen zu können. Für die Likert-skalierten Items, z.B. zur Wichtigkeit bestimmter Themen für die Zukunft der Versorgungsforschung in Bayern, wurden darüber hinaus Mittelwerte berechnet. Die Auswertung der offenen Fragen bzw. Freitextangaben basierte auf dem Verfahren der quantitativen Inhaltsanalyse [13].

Die Auswertung der geschlossenen Fragen wurden mit Stata/SE, Version 12.1, durchgeführt.

\section{Ergebnisse}

27 von insgesamt 36 LAGeV-Mitgliedspersonen nahmen an der Befragung teil, was einer Rücklaufquote von 75,0\% entspricht ( $\bullet$ Tab. 2). Etwas mehr als die Hälfte der Befragten zählen zur Gruppe „Hochschulen, Forschungsinstitute“, darunter 7 Vertreter aus medizinischen Fakultäten sowie 4 Gesundheitsökonomen. Jeweils 3 bzw. 4 Befragte gehören zu den Gruppen „Leistungserbringer, Kassenärztliche Vereinigung, Kammern, Bayerische Krankenhausgesellschaft“, „Sozialversicherungsträger“ bzw. „Ministerien“.

Tab. 2 Response der Befragung der Bayerischen Landesarbeitsgemeinschaft Gesundheitsversorgungsforschung (LAGeV).

\begin{tabular}{llll}
\hline Akteursgruppe & Befragte & $\begin{array}{l}\text { Erfolgreich } \\
\text { Befragte }\end{array}$ & $\begin{array}{l}\text { Response } \\
\text { (in \%) }\end{array}$ \\
$\begin{array}{l}\text { Hochschulen, Forschungs- } \\
\text { institute }\end{array}$ & 16 & 15 & 93,8 \\
$\begin{array}{l}\text { Leistungserbringer, KV, } \\
\text { Kammern, BKG }\end{array}$ & 6 & 4 & 66,7 \\
\hline $\begin{array}{l}\text { Sozialversicherungsträger } \\
\text { Ministerien }\end{array}$ & 4 & 3 & 75,0 \\
\hline Sonstige & 6 & 3 & 50,0 \\
\hline Gesamt & 4 & 2 & 50,0 \\
\hline & 36 & 27 & 75,0 \\
\hline
\end{tabular}

\section{Stand der Versorgungsforschung in Bayern}

Die Bestandsaufnahme im ersten Teil der Befragung fokussiert die Zufriedenheit der Akteure mit wesentlichen Aspekten der Versorgungsforschung in Bayern. Wie die $\bullet$ Abb. 1 demonstriert, sind die Zufriedenheitswerte auf der Skala von 0 bis 10 Punkten insgesamt eher niedrig. Die geringste Zufriedenheit herrscht mit der Wirksamkeit der Versorgungsforschung in der Politikberatung mit einem Mittelwert von 2,38 Punkten vor. Etwas weniger unzufrieden sind die Befragten mit dem Output (Tagungen, Publikationen, Zitierhäufigkeit usw.) in der Versorgungsforschung mit einem mittleren Punktwert von 3,29. Noch am ehesten zufrieden zeigen sich die Befragten mit den Rahmenbedingungen mit durchschnittlich 3,79 Punkten. Bei der nach Akteursgruppen stratifizierten Analyse in $\bullet$ Abb. 1 ergeben sich deutliche Unterschiede. So beurteilen die Vertreter von Ministerien die untersuchten Aspekte wesentlich besser als der Durchschnitt. Die Hochschulen und Forschungsinstitute sowie die Gruppe der Leistungserbringer sind dagegen besonders unzufrieden mit den Rahmenbedingungen und der Wirksamkeit in der Politikberatung. Die Sozialversicherungsträger äußern sich zu den Rahmenbedingungen ähnlich zufrieden wie die ministeriellen Vertreter, sind aber am unzufriedensten von allen Akteursgruppen mit dem Output der Versorgungsforschung und der Wirksamkeit in der Politikberatung. Auf die offene Frage nach bisherigen fördernden Faktoren für die Versorgungsforschung in Bayern antworten 15 Befragte (55,6\%). Die Freitextangaben zu den fördernden Faktoren lassen sich wie folgt kategorisieren und nach der Zahl der Nennungen in eine Rangfolge bringen:

1. gute vorhandene (Forschungs-)Infrastruktur,

2. Aktivitäten des Landes,

3. finanzielle Forschungsförderung und

4. diverse weitere Förderfaktoren.

Die gute vorhandene (Forschungs-)Infrastruktur wird vor allem von Vertretern der medizinischen Fakultäten und Gesundheitsökonomen sowie von den Vertretern der Ministerien als fördernd für die bisherige Versorgungsforschung in Bayern beurteilt. Über bisherige hemmende Faktoren für die Versorgungsforschung in Bayern geben 19 der Interviewpersonen (70,4\%) Auskunft. Nachfolgend werden die Antwortkategorien nach Zahl der Nennungen aufgelistet:

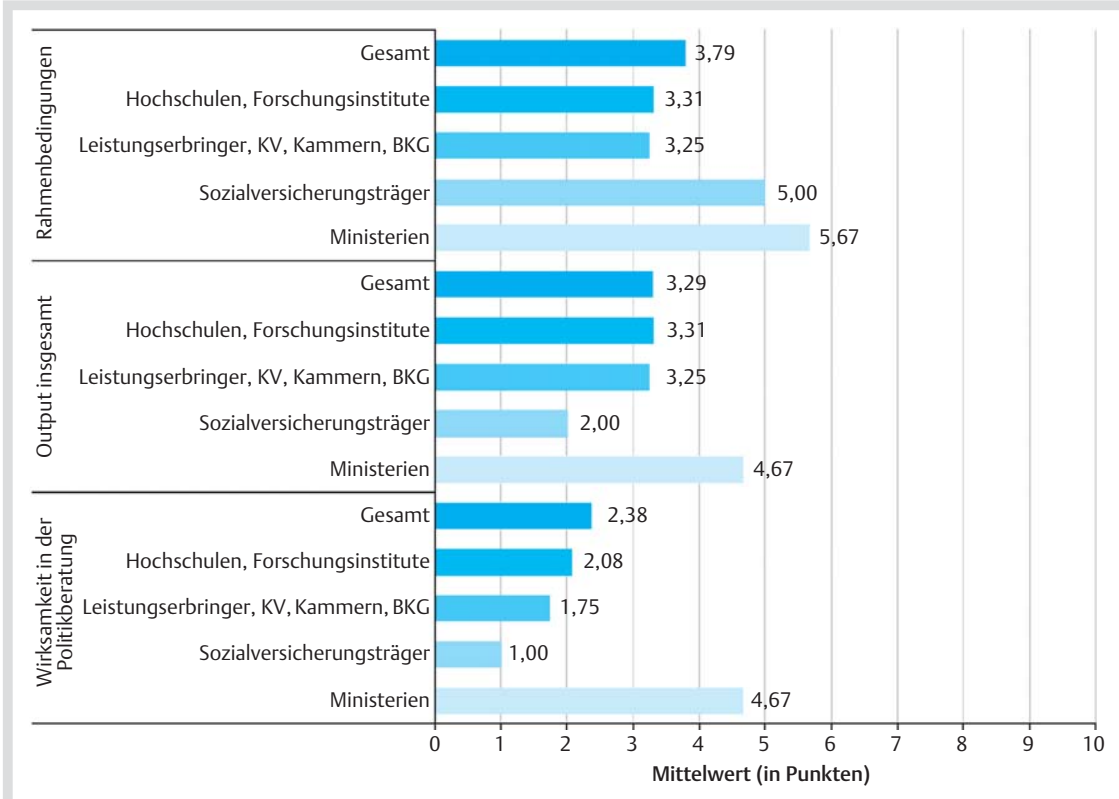

Abb. 1 Zufriedenheit der Mitglieder der Bayerischen Landesarbeitsgemeinschaft Gesundheitsversorgungsforschung (LAGeV) mit zentralen Aspekten der Versorgungsforschung in Bayern. Mittelwerte basieren auf einer 11-stufigen Likert-Skala von 0 (ganz und gar unzufrieden) bis 10 (ganz und gar zufrieden). Quelle: eigene Abbildung. 


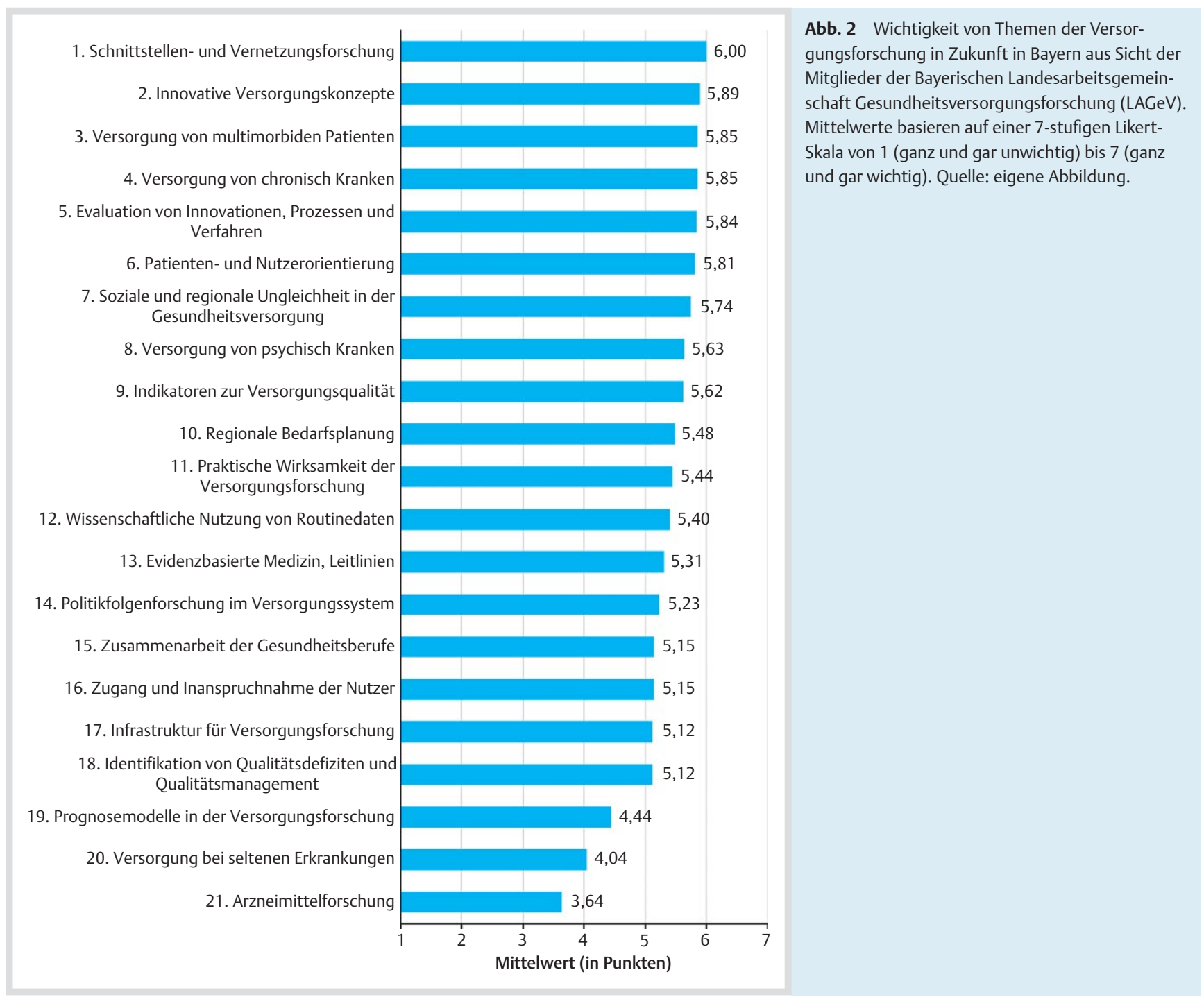

1. Fehlen von Koordination, Vernetzung und Rahmenkonzept,

2. konkurrierende Interessen,

3. Unterfinanzierung,

4. schwache universitäre Infrastruktur,

5. Datenschutz und Datennutzung sowie

6. diverse weitere Hemmfaktoren.

Während das Fehlen von Koordination, Vernetzung und Rahmenkonzept fast ausschließlich von der Seite der Wissenschaftler beklagt wird, werden konkurrierende Interessen von allen Akteursgruppen thematisiert. Das Problem der Unterfinanzierung beschreiben alle Akteursgruppen mit Ausnahme der Leistungserbringer und Sozialversicherungsträger. Eine schwache universitäre Basis wird seitens der Gesundheitsökonomie, Fachhochschulen und Sonstige, nicht aber von den Vertretern der medizinischen Fakultäten erwähnt.

\section{Zukunftsthemen und Potenziale der Versorgungsforschung in Bayern}

Das Thema „Schnittstellen- und Vernetzungsforschung“ wird von den Interviewten für die Versorgungsforschung in Bayern als am wichtigsten für die Zukunft eingeschätzt (Mittelwert (MW) 6,00 auf einer Skala von 1 bis 7). Wie die Rangliste nach Wichtigkeit in $\bullet$ Abb. 2 zeigt, folgen mit geringem Abstand die Zukunftsthemen „Innovative Versorgungskonzepte“ (MW 5,89), „Versorgung von multimorbiden Patienten“, „Versorgung von chronisch Kranken“ (beide MW 5,85) und „Evaluation von Innovationen, Prozessen und Verfahren“ (MW 5,84) ( $\bullet$ Abb. 2). Auch bei den nachfolgenden Zukunftsthemen, z.B. „Patienten- und Nutzerorientierung“ (MW 5,81) sowie „Soziale und regionale Ungleichheit in der Versorgung“ (MW 5,74) bestehen nur graduelle Unterschiede. Hier ist von einer im Wesentlichen gleichrangigen Bedeutung der genannten Themen auszugehen.

Jedoch fällt die Bewertung einiger Themen am Ende der Liste deutlich ab. So werden die Themen „Arzneimittelforschung“ (MW 3,64), „Versorgung bei seltenen Erkrankungen“ (MW 4,04) und „Prognosemodelle in der Versorgungsforschung“ (MW 4,44) als relativ unwichtig beurteilt ( $\bullet$ Abb. 2).

Die Wichtigkeit von einzelnen Zukunftsthemen wird aber in der Perspektive der einzelnen Akteursgruppen unterschiedlich eingeschätzt. Stratifiziert man die Analyse nach Akteursgruppen, verschieben sich die Rangfolgen. Während in der Gruppe „Hochschulen, Forschungsinstitute“ das Thema „Evaluation von Innovationen, Prozessen und Verfahren“ den ersten Platz belegt, favorisieren die Gruppe „Leistungserbringer, KV, Kammern, BKG“ das Thema „Regionale Bedarfsplanung“, die Gruppe „Sozialversicherungsträger“ das Thema „Schnittstellen- und Vernetzungsforschung“ und die Gruppe „Ministerien“ gleichermaßen die Themen „Innovative Versorgungskonzepte“, „Evidenzbasierte Medizin, Leitlinien“ sowie „Praktische Wirksamkeit der Versorgungsforschung“ (nicht dargestellt). 
Konkrete Ansatzpunkte zur Weiterentwicklung der Versorgungsforschung in Bayern werden von 24 befragten Personen $(88,9 \%)$ benannt. Die folgende Liste informiert über die Ansätze nach Zahl der Nennungen:

1. Rahmenkonzeption und gezielte Forschungsförderung,

2. Vernetzung und Kooperation,

3. Forschungsfinanzierung,

4. Schnittstelle Politik-Versorgungsforschung,

5. Einrichtung von Lehrstühlen,

6. Datennutzung und -zusammenführung sowie

7. diverse weitere Ansatzpunkte.

Von allen Akteursgruppen wird der Vorschlag einer Rahmenkonzeption und gezielten Forschungsförderung sowie einer entsprechenden Forschungsfinanzierung geteilt. Darunter fallen Aspekte wie „Koordination von Fördermaßnahmen“, „Aufbau eines Forschungstopfes“ und „Finanzierung von Forschung(sverbünden) für prioritäre Themen“. Die hohe Priorität ist auch Ausdruck dessen, dass das Fehlen von Koordination, Vernetzung und Rahmenkonzept sowie eine Unterfinanzierung bisher als Haupthemmnisse für die Versorgungsforschung in Bayern beschrieben werden. Die Befragten verbinden damit zudem die Chance auf einen verbesserten Ressourceneinsatz. Vernetzung und Kooperation als zweithäufigste Kategorie wird vorwiegend vonseiten der Wissenschaft als ein Ansatzpunkt zur Weiterentwicklung der Versorgungsforschung in Bayern beschrieben. Als Chancen einer stärkeren Vernetzung und Kooperation beschreiben einige Befragte die LAGeV als neue Struktur sowie die Möglichkeit einer stärkeren Zusammenarbeit. Hinsichtlich der Schnittstelle Politik-Versorgungsforschung werden u.a. „regelmäßige Information der Staatsregierung über Ergebnisse der Versorgungsforschung“ und „Ableitung von Handlungsempfehlungen (Politikberatung)“ genannt, um eine „konstruktive Einflussnahme auf politische Entscheidungen“ zu ermöglichen. Vermieden werden sollten dagegen „Doppelaktivitäten (Bund/Land)“ und eine „Orientierung an der „deutschen“ Versorgungsforschung“.

22 bzw. 20 der Befragten (81,5\% bzw. 74,1\%) formulieren Chancen und Risiken für die Versorgungsforschung in Bayern. Die weitaus häufigste Zahl an Nennungen bezieht sich auf die Optimierung der Versorgung, z. B. „optimale Behandlung von Patienten“, „unabhängige Analyse der med. Versorgung“, „Aufdeckung von Versorgungsdefiziten“ und „proaktive Entwicklung innovativer Versorgungskonzepte“. Einige Chancen wie die Verbesserung der Schnittstelle Politik-Versorgungsforschung, der verbesserte Ressourceneinsatz, die LAGeV als neue Struktur und eine stärkere Zusammenarbeit wurden bereits an anderer Stelle genannt. Weitere Kategorien sind die Versorgungsforschung als Innovationsmotor für die Gesundheitswirtschaft sowie die Stärkung der Versorgungsforschung.

Als Risiken der Versorgungsforschung in Bayern werden hauptsächlich „Eigeninteressen, politische Einflussnahme und Lobbyismus“ sowie „Unterfinanzierung“ angegeben. Beides würde ein Fortdauern der bereits beschriebenen hemmenden Faktoren (konkurrierende Interessen, Unterfinanzierung) bedeuten, d. h. falls die Ansatzpunkte für die Weiterentwicklung der Versorgungsforschung ungenutzt bleiben. Das Risiko von Eigeninteressen, politischer Einflussnahme und Lobbyismus wird durchweg von allen Akteursgruppen gesehen - mit Ausnahme der Befragten aus medizinischen Fakultäten. Letztere nennen lediglich Aspekte der Unterfinanzierung. Als weitere Risiken werden „Dominanz der Medizin“ und „unzureichende Abstimmung Bund/Land“ antizipiert.

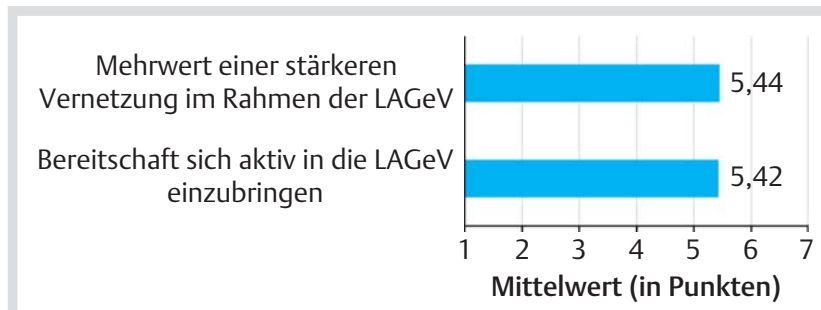

Abb. 3 Mehrwert einer stärkeren Vernetzung im Rahmen der Bayerischen Landesarbeitsgemeinschaft Gesundheitsversorgungsforschung (LAGeV) aus Sicht ihrer Mitglieder und deren Bereitschaft zur aktiven Mitarbeit. Mittelwerte basieren auf einer 7-stufigen Likert-Skala von 1 (ganz und gar gering) bis 7 (ganz und gar hoch). Quelle: eigene Abbildung.

\section{Konkrete Kooperation in der LAGeV}

Den Mehrwert einer stärkeren Vernetzung im Rahmen der LA$\mathrm{GeV}$ sowie die eigene Bereitschaft, sich aktiv in die LAGeV einzubringen, bewerten die Interviewpersonen gemäß $\bullet$ Abb. 3 eher hoch (MW 5,44 bzw. 5,42 auf einer Skala von 1 bis 7). Die höchsten Werte sind dabei tendenziell in der Gruppe „Hochschulen, Forschungsinstitute“ zu beobachten. Bei der Art der gewünschten Mitarbeit stimmen die Befragten am häufigsten der „Beteiligung an Kooperationsprojekten“ (70,4\%) sowie der „Mitarbeit in themenbezogenen Arbeitsgruppen“ (63,0\%) zu.

\section{Diskussion}

$\nabla$

Als ein Hauptergebnis ergibt die Befragung, dass die Zufriedenheit der Akteure mit dem Stand der Versorgungsforschung in Bayern relativ gering ist. Besonders gering ist die Zufriedenheit hinsichtlich der Wirksamkeit in der Politikberatung. Die Erhebung zeigt auf, dass zwischen den Akteursgruppen aus Wissenschaft, Politik und Praxis divergierende Einschätzungen zum Stand der Versorgungsforschung, zu Zukunftsthemen, Förderund Hemmfaktoren und Ansatzpunkte vorliegen.

Die gute vorhandene (Forschungs-)Infrastruktur und die Aktivitäten des Landes Bayern werden von den Befragten zwar gewürdigt, sie bemängeln an der bisherigen Versorgungsforschung aber Erschwernisse durch das Fehlen von Koordination, Vernetzung und Rahmenkonzeption sowie konkurrierende Interessen und Unterfinanzierung. Auffällig ist dabei, dass die Vertreter medizinischer Fakultäten und zum Teil auch des Faches Gesundheitsökonomie die gute vorhandene (Forschungs-)Infrastruktur als einen Hauptförderfaktor benennen, während die übrigen Wissenschaftler in der schwachen universitären Infrastruktur ein Haupthemmnis der Versorgungsforschung in Bayern sehen. Diese Differenz könnte ein Hinweis auf die ungleich verteilten Ressourcen und Durchsetzungsfähigkeit verschiedener Disziplinen bzw. Institutionen sein.

Ansatzpunkte zur Weiterentwicklung der Versorgungsforschung in Bayern bestehen aus Sicht der Befragten vor allem in der Beseitigung der bisher hemmenden Faktoren. Dazu würden eine Rahmenkonzeption und gezielte Forschungsförderung, Vernetzung und Kooperation, Forschungsfinanzierung sowie eine Verbesserung der Schnittstelle Politik-Versorgungsforschung beitragen. Ein weiterer Ansatzpunkt wird in einer stärkeren Vernetzung und Kooperation erkannt. Das korrespondiert mit Bewertungen der Befragten zur LAGeV. Der Mehrwert einer stärkeren Vernetzung im Rahmen der LAGeV wird als eher hoch eingeschätzt; 
Gleiches gilt für die Bereitschaft der Mitglieder, insbesondere aus der Wissenschaft, sich aktiv in die LAGeV einzubringen.

Die vorliegende Untersuchung bestätigt die allgemeine Feststellung von Pfaff und Schrappe [7], wonach die Akteure der Versorgungsforschung zersplittert sind und die Finanzierung ungenügend ist. Das Fehlen von Koordination, Vernetzung und Rahmenkonzept sowie die Unterfinanzierung werden in der Befragung als 2 der bisherigen Haupthemmnisse der Versorgungsforschung in Bayern identifiziert. Insofern gilt der gesamtdeutsche Befund auch für das Bundesland Bayern. Weiterhin bestätigen die in dieser Untersuchung identifizierten Ansatzpunkte zur Weiterentwicklung der Versorgungsforschung in Bayern die von Pfaff und Schrappe [7] festgestellte Notwendigkeit von Koordination und Netzwerkbildung in überregionalen Strukturen, auch außerhalb von Universitäten.

Für die Zukunft der Versorgungsforschung in Bayern wird das Thema Schnittstellen- und Vernetzungsforschung als am wichtigsten bewertet, gefolgt von den Themen innovative Versorgungskonzepte, Versorgung von multimorbiden Patienten bzw. chronisch Kranken sowie Evaluation von Innovationen, Prozessen und Verfahren. Allerdings zeigen sich erhebliche Unterschiede zwischen den Akteursgruppen, die in den jeweils spezifischen Interessen und Aufgaben der verschiedenen Akteure begründet sein dürften. Bezüglich der für die Versorgungsforschung wichtigen Zukunftsthemen weisen die von Grenz-Fahrenholtz et al. [9] identifizierten wichtigen Themen(cluster) und die Themenrangfolge auf Basis der vorliegenden Befragung große Gemeinsamkeiten auf. In beiden Untersuchungen wird das Thema sektorenübergreifende Versorgung bzw. Schnittstellen- und Vernetzungsforschung an erste Stelle gesetzt. Weitere Themen mit ähnlich hoher Priorisierung sind die Evaluation von Innovationen, Prozessen und Verfahren (vgl. auch SVR [1]), Patienten- und Nutzerorientierung, die Versorgung von chronisch Kranken sowie soziale und regionale Ungleichheit in der Versorgung.

Die Stärke der vorliegenden Untersuchung liegt zum einen in der Befragung aller an der Gesundheitsversorgung sowie der Versorgungsforschung in Bayern beteiligter Akteursgruppen. Neben Vertretern der Wissenschaft, Leistungserbringer und Sozialversicherungsträger wurden auch Vertreter der Ministerien und weiterer Einrichtungen befragt. Zum anderen erzielte die Befragung eine sehr hohe Rücklaufquote und die untersuchten Akteursgruppen (und damit Perspektiven auf die Versorgungsforschung) sind mit jeweils mehreren zurückgesandten Fragebögen in die Datenauswertung eingegangen.

Einschränkend ist anzumerken, dass die berichteten Ergebnisse auf einer kleinen Fallzahl beruhen. Dies gilt insbesondere für die untersuchten Subgruppen wie die Sozialversicherungsträger und Ministerien, weswegen auf statistische Tests und die Berechnung von Konfidenzintervallen verzichtet wurde. Daher sind bspw. die berichteten Gruppenunterschiede bei der Zufriedenheit mit dem Stand der Versorgungsforschung auch als Hinweise zu betrachten. Weiterhin wurden die Antworten der verschiedenen Akteursgruppen nicht gewichtet, sodass die Gruppe mit der höchsten Zahl an Befragten auch den größten Einfluss auf die dargestellten Mittelwerte hat.

\section{Schlussfolgerungen}

Die vorliegende Bestandsanalyse der Versorgungsforschung in Bayern unterstreicht die Notwendigkeit einer konzertierten Weiterentwicklung der Versorgungsforschung mit allen relevanten Akteuren, wie sie in der Bayerischen Landesarbeitsge- meinschaft Gesundheitsversorgungsforschung (LAGeV) bereits angelegt ist. Sie zeigt die Potenziale einer solchen Kooperation in Hinblick auf Koordination, Vernetzung, Rahmenkonzeption und Priorisierung von Themen auf. Zentrale Herausforderungen dürften die Ermittlung des Bedarfs von Politikberatung, die Berücksichtigung von Ergebnissen der Versorgungsforschung im politischen Handeln sowie die stärkere Bearbeitung von politikrelevanten Problemen in der Versorgungsforschung darstellen.

Die Befunde dieser Befragung können für eine Weiterentwicklung der Versorgungsforschung in Bayern und darüber hinaus genutzt werden. Der Erfolg wird wesentlich davon abhängen, ob es gelingt, die verschiedenen Fach-, Handlungs- und Zeitlogiken in Wissenschaft, Politik und Praxis zu integrieren.

\section{Danksagung \\ $\nabla$}

Die Autoren bedanken sich bei den Mitgliedern der Bayerischen Landesarbeitsgemeinschaft Gesundheitsversorgungsforschung (LAGeV), die an der Befragung teilgenommen haben.

Interessenkonflikt: Die Autoren geben an, dass kein Interessenkonflikt besteht.

\section{Literatur}

1 SVR. Sondergutachten 2012 des Sachverständigenrates zur Begutachtung der Entwicklung im Gesundheitswesen. Wettbewerb an der Schnittstelle zwischen ambulanter und stationärer Gesundheitsversorgung. Bundestag Drucksache 17/10323. 2012

2 SVR. Gutachten 2000/2001 des Sachverständigenrates für die Konzertierte Aktion im Gesundheitswesen. Bedarfsgerechtigkeit und Wirtschaftlichkeit. Band II: Qualitätsentwicklung in Medizin und Pflege. Bundestag Drucksache 14/5661. 2001

3 SVR. Gutachten 2009 des Sachverständigenrates zur Begutachtung der Entwicklung im Gesundheitswesen. Koordination und Integration - Gesundheitsversorgung in einer Gesellschaft des längeren Lebens. Bundestag Drucksache 16/13770. 2009

4 Raspe $H$, Pfaff $H$, Härter $M$ et al. Versorgungsforschung in Deutschland: Stand - Perspektiven - Förderung. Bonn: Deutsche Forschungsgemeinschaft; 2010

5 Lohr KN, Steinwachs DM. Health services research: an evolving definition of the field. Health Services Research 2002; 37: 7-9

6 Pfaff $H$. Versorgungsforschung - Begriffsbestimmung, Gegenstand und Aufgaben. In: Pfaff H, Schrappe M, Lauterbach K et al. (Hrsg.) Gesundheitsversorgung und Disease Management: Grundlagen und Anwendungen der Versorgungsforschung. Bern: Verlag Hans Huber; 2003; 13-23

7 Pfaff $H$, Schrappe M. Einführung in die Versorgungsforschung. In: Pfaff $\mathrm{H}$, Neugebauer EA, Glaeske G et al. (Hrsg.) Lehrbuch Versorgungsforschung Systematik - Methoden - Anwendung. Stuttgart: Schattauer; 2011; 1-39

8 Bayerischer Landtag. Beschluss des Bayerischen Landtags „Versorgungsforschung im Freistaat Bayern weiterentwickeln“, Drucksache 16/8005. 2011

9 Grenz-Farenholtz B, Schmidt A, Verheyen F et al. Zukunftsthemen der Versorgungsforschung - Was meinen die Experten? Gesundheitswesen 2012; 74: 605-611

10 Glaeske G, Augustin M, Abholz $H$ et al. Epidemiologische Methoden für die Versorgungsforschung. Gesundheitswesen 2009; 71: 685-693

11 Pfaff H, Kaiser C. Aufgabenverständnis und Entwicklungsstand der Versorgungsforschung. Ein Vergleich zwischen den USA, Großbritannien, Australien und Deutschland. Bundesgesundheitsblatt Gesundheitsforschung Gesundheitsschutz 2006; 49: 111-119

12 Schubert I, Köster I, Küpper-Nybelen J et al. Versorgungsforschung mit GKV-Routinedaten. Nutzungsmöglichkeiten versichertenbezogener Krankenkassendaten für Fragestellungen der Versorgungsforschung. Bundesgesundheitsblatt Gesundheitsforschung Gesundheitsschutz 2008; 51: 1095-1105

13 Bortz J, Döring N. Forschungsmethoden und Evaluation für Humanund Sozialwissenschaftler. Heidelberg: Springer Medizin Verlag; 2005 